\title{
Infrared Scene Projector Digital Model Development
}

\author{
Mark A. Manzardo ${ }^{1}$, Brett Gossage ${ }^{1}$, J. Brent Spears ${ }^{1}$, and Kenneth G. LeSueur ${ }^{2}$ \\ ${ }^{1} 555$ Sparkman Drive, Executive Plaza, Suite 1622 \\ Huntsville, AL 35816 \\ \{mmanzardo, bgossage, bspears\}@rttc.army.mil \\ ${ }^{2}$ US Army, Developmental Test Command \\ Redstone Technical Test Center \\ CSTE-DTC-RT-E-SA, Bldg. 4500 \\ Redstone Arsenal, AL 35898-8052 \\ klesueur@rttc.army.mil
}

\begin{abstract}
This paper describes the development of an Infrared Scene Projector Digital Model (IDM). The IDM is a product being developed for the Common High Performance Computing Software Support Initiative (CHSSI) program under the Integrated Modeling and Test Environments (IMT) Computational Technology Area (CTA). The primary purpose of the IDM is to provide a software model of an Infrared Scene Projector (IRSP). Initial utilization of the IDM will focus on developing non-uniformity correction algorithms, which will be implemented to correct the inherent non-uniformity of resistor array based IRSPs. After describing the basic components of an IRSP, emphasis is placed on implementing the IDM. Example processed imagery will be included, as well.
\end{abstract}

\section{General Description of an Infrared Scene Projector (IRSP)}

In general, an IRSP can be broken down into the interaction between the following subsystems/components: a Software Control Subsystem (SCS), a Computer Image Generator Subsystem (CIGS), a Control Electronics Subsystem (CES), an Environmental Conditioning Subsystem (ECS), an Infrared Emission Subsystem (IRES), a Projection Optics Subsystem (POS), a Non-Uniformity Correction Subsystem (NUCS), a Mounting Platform Subsystem (MPS) and a System Under Test (SUT). Figure 1 provides an overview block diagram illustration of an IRSP. This figure also illustrates the typical interaction between the subsystems. Not all of these interactions are required; some are for ease of use, configuration management, system safety, or other specific needs. It is unlikely that any given IRSP utilizes all of the illustrated interfaces.

V.N. Alexandrov et al. (Eds.): ICCS 2001, LNCS 2074, pp. 531-539, 2001.

(c) Springer-Verlag Berlin Heidelberg 2001 


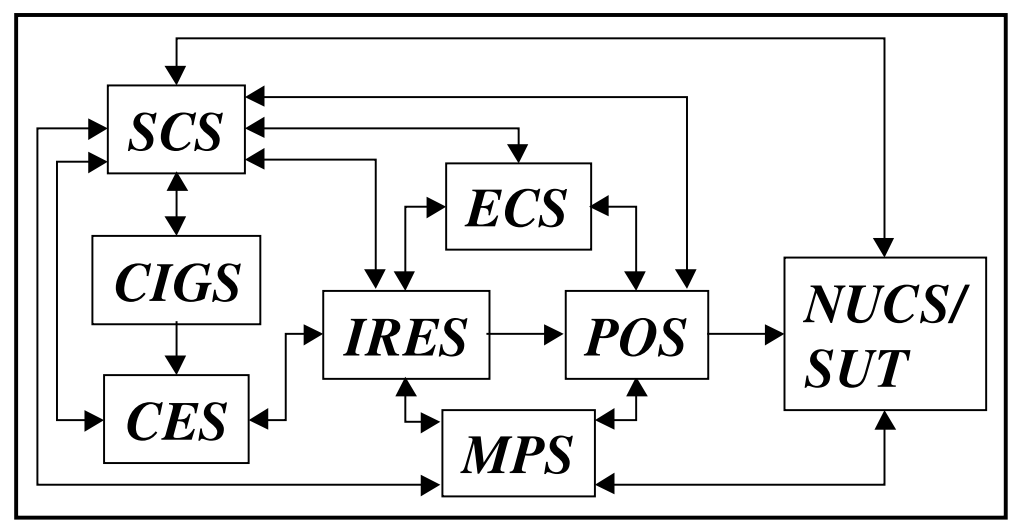

Fig. 1. Typical IRSP Block Diagram.

The following sections provide a brief description of each of the IRSP subsystems.

\subsection{Software Control Subsystem (SCS)}

The SCS can be one integrated program controlling all aspects of the IRSP from a single Graphical User Interface (GUI). The SCS can also have communication interfaces with other programs that are driving the overall simulation. The SCS can be hosted entirely on the CIGS, CES or it's own separate platform. In general, SCS components can be distributed throughout other IRSP subsystems. The form the SCS takes is usually driven by IRSP usage requirements tempered by cost restraints.

\subsection{Computer Image Generator Subsystem (CIGS)}

The CIGS is the hardware platform responsible for generating the dynamic input imagery used by the IRSP CES. The generation of this imagery is typically performed in real-time using 3-D target/terrain database(s), material database(s), etc. The result of the scene generation software (an IRSP SCS component) is a sequence of 2-D rendered scene images. The scene generator can either render these images in real-time for input to the IRSP CES or scene generation can be an initial step preparing the imagery for pre-projection processing. One such pre-projection processing step would be to perform software Real-time Non-Uniformity Correction (RNUC) on each image pixel. In some cases the scene generator is used to render extremely high fidelity scenes in non-real-time for storage and subsequent real-time playback. 


\subsection{Control Electronics Subsystem (CES)}

The CES is used to provide all the necessary analog and digital signals to drive the resistor array. In addition to the image data these signals will also typically include bias, power, and address lines. In some cases, such as test patterns, image data can be generated on the CES hardware or uploaded across the bus interface of the CES hardware (e.g., VME, VXI, ...). However, more practical applications that require real-time dynamic image sequences use a high speed digital image interface (e.g., the SGI DD02). In some cases, pipeline image processing hardware is incorporated into the CES to simulate effects not incorporated in the scene generator and/or to correct for overall IRSP simulation latency. Examples of effects not incorporated in the scene generator that can be accommodated by dedicated hardware are atmospheric blur, missile seeker focus upon closure to a target, and RNUC of the resistor array. With or without dedicated hardware to perform the above special effects, the CES can impose lossy noise or digitization effects on the image data. The output of the CES is typically either high speed digital image data over a fiber optic interface or analog voltages from high speed digital to analog converters.

\subsection{Environmental Conditioning Subsystem (ECS)}

The ECS has the primary responsibility of providing an environment for proper operation of the resistor array. The ECS is responsible for creating (and sometimes maintaining) a vacuum environment around the array. The ECS is also responsible for creating and maintaining an operating temperature for the array. In addition, in some cases, the ECS is also extended to provide thermal conditioning of other IRSP components (e.g., the POS, NUCS and/or the SUT).

The ECS typically utilizes a contaminant free vacuum pump capable of pulling a vacuum less than 3E-04 torr. A turbo-molecular vacuum pumping station is typically used to create the vacuum. In some cases, the vacuum pump remains on continuously to maintain the vacuum environment. In other cases, the IRES has the ability to maintain a vacuum level for a sufficient amount of time for operation; the IRES is sometimes assisted by a small ION pump. The array's radiometric output is known to be a function of the vacuum level. This is primarily due to allowing a convection heat dissipation path. In an absolute vacuum, the array can only dissipate heat radiatively and conductively through it's legs to the substrate. When the vacuum pressure increases, providing for a convection heat dissipation path, the resistor does not heat up as much and the radiated energy is subsequently reduced. Studies indicate that a vacuum level below 3E-04 torr is sufficient to dramatically mitigate this effect.

Due to the large substrate heat dissipation requirements of micro-resistor arrays (typically in excess of 100 Watts), the heat removal is accomplished with a fluid recirculating chiller. The operating temperature of the resistor array affects the array's radiometric output. This directly affects the obtainable background radiometric floor and stability of the IRSP. In addition, the CMOS drive substrate changes it's characteristics as a function of temperature. 


\subsection{Infrared Emission Subsystem (IRES)}

The IRES has the primary function of mounting the resistor array to allow it to interface to the rest of the IRSP. Usually, the IRES is a self-contained package providing electrical, mechanical, fluid, and vacuum interfaces. In some cases, the heat sink is external to the vacuum environment of the array, limiting the array operating temperature in this stand-alone package to something above the ambient dew point. Other arrays, are packaged in a full dewar configuration allowing the array operating temperature to go well below the dew point (some are designed to go to $-35^{\circ} \mathrm{C}$ and others can go to $20 \mathrm{~K}$ ). In some configurations, the vacuum environment not only encloses the array, but also the POS and sometimes a detector array.

In any event, the primary component of the IRES is the resistor array itself. Some examples of characteristics, which are known to affect the array's input to output response are: material type, leg length, fill factor, etc. However, non-uniformities in resistor responses occur because of manufacturing inconsistencies and defects.

There are at least a couple of known scene based effects with some resistor arrays. The first is local heating near contiguously driven regions on the array. Another is an effect termed "bus-bar-robbing" which results from voltage drops by driving a large percentage of the array.

In addition to the scene based effects there are at least a couple of transient temporal effects. One is based on the real response time of the resistor. A similar effect is intra-frame droop.

\subsection{Projection Optics Subsystem (POS)}

The POS has the responsibility to properly project the IRES radiation into a Sensor or System Under Test (SUT). This is typically accomplished by collimating the IRES radiation. The focal length and pupil of the POS is chosen to allow for valid effective SUT evaluation. Being a real optical system, the POS will have spectral transmission losses, impose geometric and diffractive blur effects and impose geometric distortion effects on the projected image.

\subsection{Non-uniformity Correction Subsystem (NUCS)}

The NUCS has the responsibility of being able to collect, reduce, and process resistor array imagery with the purpose of determining the proper correction coefficients necessary to remove the array non-uniformity's. The process has been implemented by isolating and measuring the output of each resistor as a function of input. This has been accomplished by commanding a sparse grid of resistors to multiple inputs and then stepping through the array until all resistors have been measured. The two main drawbacks to this process is the data collection time and the fact that the resistor arrays do not respond the same when commanded with a sparse grid pattern as they do when commanded with realistic scene imagery (local heating and what has been termed bus-bar robbing are considered to be the primary sources for the differences). 
Some attempts have been made to drive contiguous blocks of resistors to collect nonuniformity data. Unfortunately, most are very dependent on optical coupling and distortion, but preliminary results are promising.

The primary component of the NUCS is the IR data collection camera. The camera acts as a transducer from IR radiation to a digital image. The camera detector(s) will have a limited resolution when digitizing the input radiation signal (e.g., 12-bit digital values representing a known max and min radiance/temperature). The primary effects the camera will impose on the input scene are spatial sampling artifacts, spatial and temporal noise, geometric distortion, and geometric and diffractive blur effects.

\subsection{Mounting Platform Subsystem (MPS)}

The MPS has the responsibility of aligning the IRES with the POS and the SUT (or NUCS camera). This can be a manual process using stages, rails, tripod(s), or equivalent. This can also be a fully computer controlled process using motorized stages and gimbals. In any event, there will always be some limited alignment error (at best on the order of the detector/resistor array spatial resolution).

\subsection{System Under Test (SUT)}

The SUT is the infrared imaging device being evaluated with the Infrared Scene Projector.

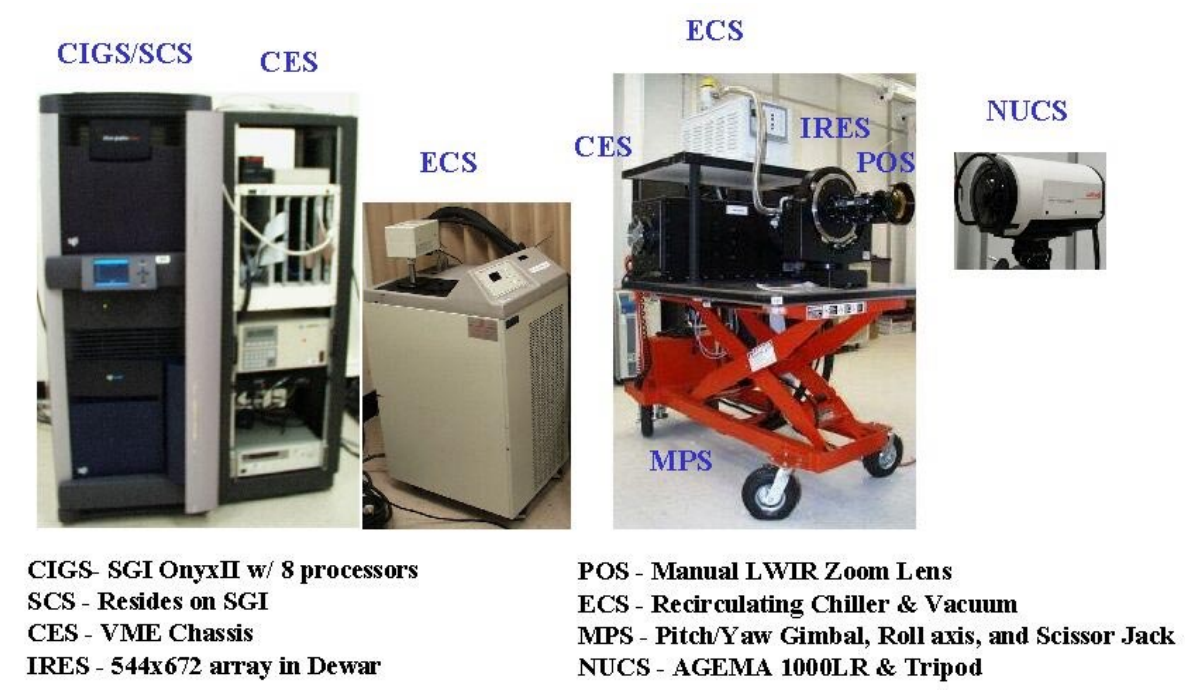

Fig. 2. DEGA System (Example IRSP). 


\subsection{Example IRSP}

For illustrative purposes, Figure 2 shows components of an example IRSP. Specifically, this is the Dynamic Infrared Scene Projector (DIRSP) Engineering Grade Array (DEGA) system used by the US Army Redstone Technical Test Center.

\section{IDM Implementation and Example Imagery}

The following sub-sections describe the simulation architecture used to implement the IDM and some example imagery. A detailed mathematical description of how the IDM is designed to accommodate all the IRSP effects is provided in a simultaneously published companion paper: "Infrared Scene Projector Digital Model Mathematical Description". In it's current state, the IDM incorporates the IRSP effects that tend to redistribute the image energy as detected by the SUT. These types of effects are: resistor fill factor, optical distortion, optical geometric aberrations, SUT diffraction, and SUT geometric aberrations. Over the next couple of years the IDM will grow to encompass the rest of the IRSP effects. However, the above effects are estimated to be the most computationally challenging, which justifies why they have been incorporated first.

\subsection{Common Simulation Framework (CSF) Simulation Architecture}

The IDM utilizes the Common Simulation Framework (CSF) to provide an IDM user with the capability to manage, store and execute the simulation. The CSF is a DoDdeveloped simulation architecture managed by US Army Aviation and Missile Research, Development and Engineering Center, Huntsville AL. The CSF-hosted IDM supports the visual assembly of component models, attribute management, and

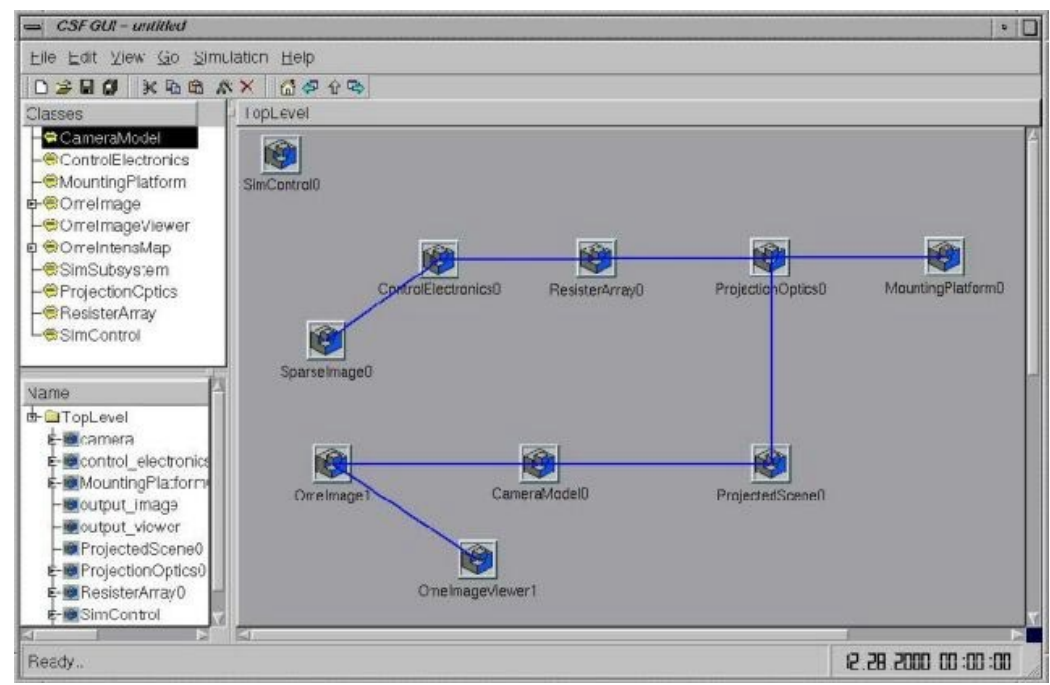

Fig. 3. CSF Implementation of the IDM. 
model execution all within a unified application. The IDM software is built on the CSF framework libraries thus providing a seamless CSF/IDM integration. Since the CSF is written entirely in $\mathrm{C}++$, run-time efficiency is maintained and multi-threading is easily applied. The object-oriented "plug-n-play" features of the CSF allow different models (e.g. cameras, resister arrays) to be substituted without changing the software. Figure 3 shows a screen-shot of the CSF-hosted IDM user interface.

In addition to using the CSF to host the IDM simulation, we have extended the CSF executive to include a real-time, frame-based scheduler. This allows the models to be executed in the same environment as the real-time NUC algorithms. The realtime executive is launched from within the CSF GUI shown above and allows a user to run, pause, reset, and stop the simulation. Pluggable image viewers are provided for viewing the results at various points in the processing chain.

\subsubsection{Parallel Speedup of IDM}

A parallel implementation of the IDM was performed for a 400(H)x200(V) resolution camera and a $672(\mathrm{H}) \times 544(\mathrm{~V})$ resolution resister array on a Silicon Graphics Incorporated (SGI) Onyx-2 with $8 \times 300 \mathrm{MHz}$ processors. Distortion effects calculations were not included in this parallel implementation. Figure 4 illustrates the comparison between the actual achieved run times versus the theoretical limit.

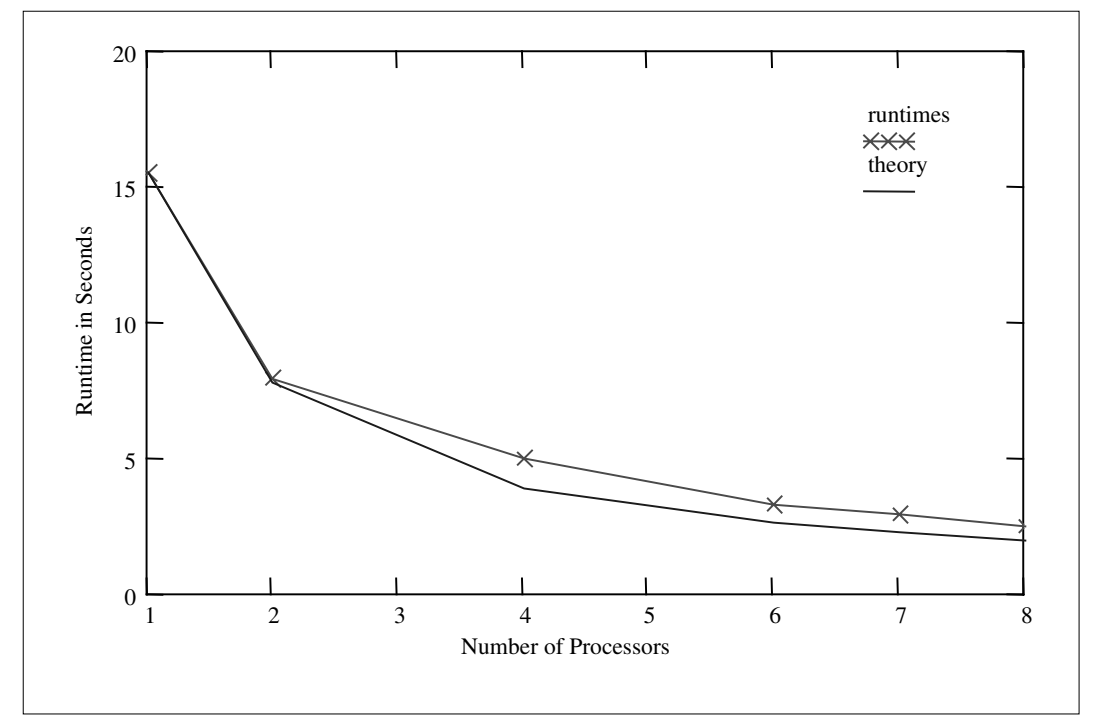

Fig. 4. Parallel Speedup of the IDM.

\subsection{Example Imagery}

The following example imagery illustrates usage of the IDM. The input represents the image driving a $672(\mathrm{H}) \times 544(\mathrm{~V})$ resistor array. The output represents the image captured by a $800(\mathrm{H}) \times 405(\mathrm{~V})$ camera. The input was a sparse resistor pattern, where 
every $16^{\text {th }}$ row and every $14^{\text {th }}$ column resistor was driven. One input image began the sparse pattern at resistor $(0,0)$ the other began the pattern at resistor $(1,1)$. Although the IDM processes the entire camera image, for illustrative purposes only a portion of the image is shown here. Figure 4 shows the upper $5 \times 5$ driven resistors.

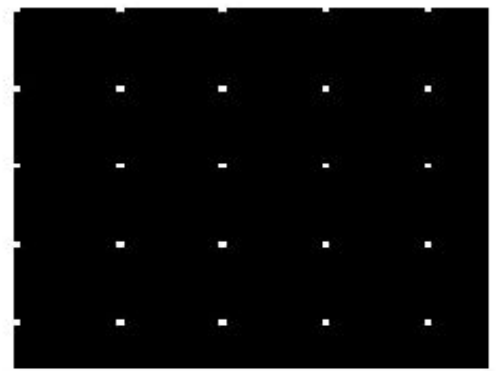

(a)

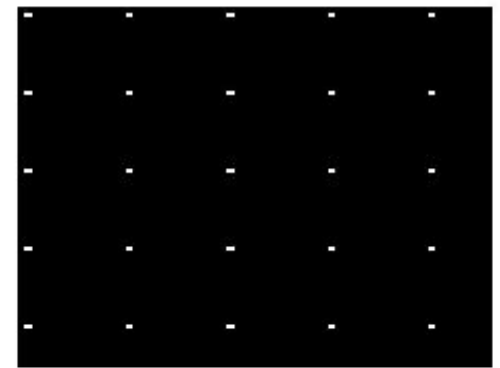

(b)

Fig. 5. 16x14 Sparse Resistor Input Images to the IDM: (a) start at $(0,0)$, (b) start at $(1,1)$.

Figure 5 illustrates the output of the IDM for the two input images with and without distortion.

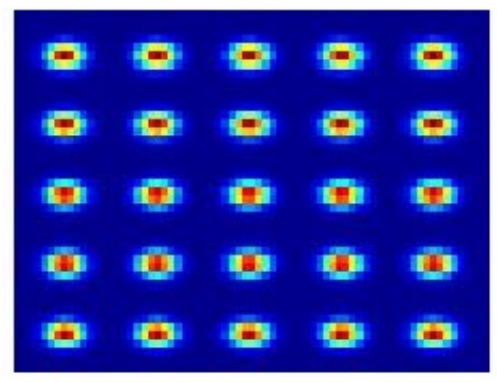

(a)

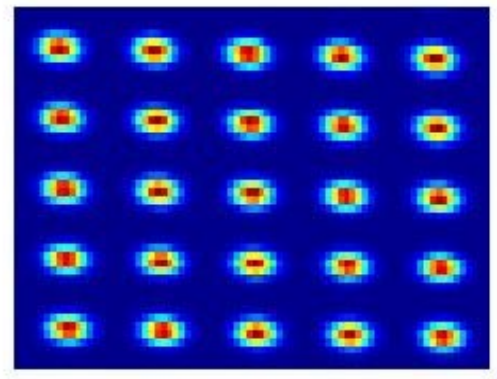

(c)

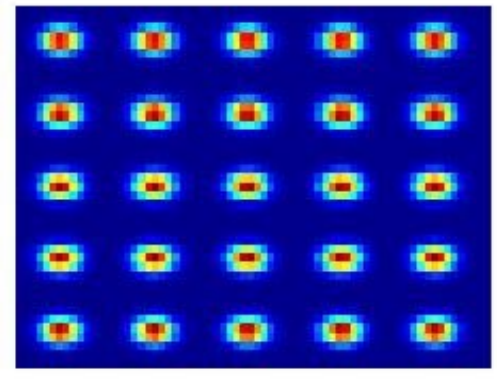

(b)

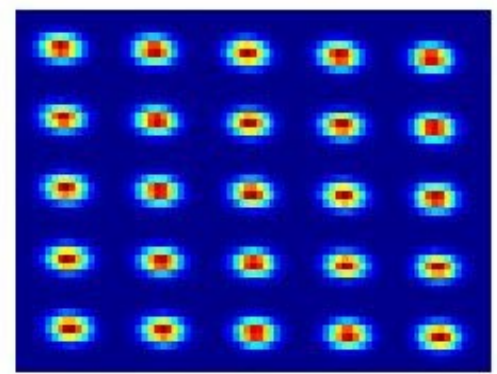

(d)

Fig. 6. Output IDM Images without (a, b) and with (c, d) Distortion. 
Of interest in the IDM output imagery is the noticeable variation of detected energy distribution from resistor to resistor. This basically illustrates why a simple single convolution kernal for the whole image would be inaccurate. In general, one way to think of the process is that each detector uses a different convolution kernal of variable size and distribution. This is necessary to account for mis-registration between the resistors and detectors, optical distortion, and walk-off registration between the resistors and detectors. Walk-off registration occurs when the pitch of the detector samples does not match the pitch of the resistors. As the output imagery illustrates, the need for a variable convolution kernal holds true even without distortion.

\section{Conclusions and Continuing Efforts}

The IDM output has been validated from a "that looks about right" perspective. The output looks very reminiscent of real collected imagery. The goal for validation of the IDM is to eventually be able to compare real versus IDM imagery using objective image statistics and comparison metrics. The validation phase can begin after incorporating the rest of the IRSP effects.

Recall that in it's current state, the IDM incorporates the IRSP effects that tend to redistribute the image energy as detected by the SUT. These types of effects are: resistor fill factor, optical distortion, optical geometric aberrations, SUT diffraction, and SUT geometric aberrations. Over the next couple of years the IDM will grow to encompass the rest of the IRSP effects.

Efforts will continue to parallelize code for use on HPC multi-processor platforms. 